\title{
Corela
}

Cognition, représentation, langage

HS-16 | 2015

Diversité des pratiques de recherche en science du langage

\section{Variation linguistique perçue : quelques localités du Piémont occidental (Italie). Questions de méthodologie en dialectologie perceptuelle}

\section{Silvia Gally}

\section{OpenEdition}

\section{Journals}

Édition électronique

URL : http://journals.openedition.org/corela/4013

DOI : 10.4000/corela.4013

ISSN : 1638-573X

Éditeur

Cercle linguistique du Centre et de l'Ouest - CerLICO

Référence électronique

Silvia Gally, «Variation linguistique perçue : quelques localités du Piémont occidental (Italie).

Questions de méthodologie en dialectologie perceptuelle », Corela [En ligne], HS-16 | 2015, mis en ligne le 29 juin 2015, consulté le 19 avril 2019. URL : http://journals.openedition.org/corela/4013 ; DOI : $10.4000 /$ corela.4013

Ce document a été généré automatiquement le 19 avril 2019

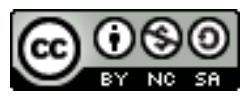

Corela - cognition, représentation, langage est mis à disposition selon les termes de la licence Creative Commons Attribution - Pas d'Utilisation Commerciale - Partage dans les Mêmes Conditions 4.0 International. 


\title{
Variation linguistique perçue : quelques localités du Piémont occidental (Italie). Questions de méthodologie en dialectologie perceptuelle
}

\author{
Silvia Gally
}

\section{Quelques définitions et explications du terme dialectologie perceptuelle}

1 Les recherches dans le domaine de la dialectologie perceptuelle (DP) sont très variées et résultent de différentes «écoles » linguistiques. Un grand nombre d'entre elles ont été réunies par le linguiste Dennis R. Preston dans un ouvrage, paru en deux volumes, et nommé Handbook of Perceptual Dialectology (Preston, 1999 ; Long \& Preston, 2003). Chronologiquement, les premières recherche dans le secteur ont vu le jour aux Pays-Bas dans les années 1930 (Goeman 1989; Preston, 1999 : 3-38; Weijnen, 2003), puis quelques décennies plus tard au Japon (Grooaters, 1959, 1964 ; Mase, 1964 ; Preston, 1999 : 39-146, 177-226). Ensuite, les études menées par Preston aux Etas-Unis (Preston, 1989 ; Niedzielski \& Preston 2000; Long \& Preston, 2003) ont suscité l'engouement des chercheurs du monde entier (pour les études menées en France, cf. Léonard, 1987, 2003 ; Putska, 2007 ; Falkert, 2010 ; pour l'Italie $c f$. Telmon, 2000 ; Cini \& Regis, 2000 ; Iannàccaro \& Dell'Aquila, 2001 ; Iannàccaro, 2000, 2002 ; Dell'Aquila \& Iannàccaro, 2006).

2 Le terme dialectologie perceptuelle, utilisé en France, a été traduit de l'anglais Perceptual Dialectology (Léonard, 1987, 2003; Putska, 2007; Falkert, 2010). Cette terminologie s'intéresse aux "mécanismes perceptuels », et ne se réfère pas aux " tests perceptifs » utilisés en phonétique (Falkert, 2010).

La DP s'intéresse à l'étude de la conscience linguistique des locuteurs. Plus précisément, on $\mathrm{y}$ analyse les perceptions et les représentations des faits linguistiques que peuvent avoir 
les locuteurs de leurs propres parlers et de ceux avec lesquels ils entrent en contact dans un espace géographique donné (Preston, 1989, 1999, 2002; Berruto, 2000 ; Telmon, 2000 ; Dell'Aquila \& Iannàccaro, 2001; Iannàccaro 2002). Ainsi, ce que les locuteurs croient et perçoivent quant aux différentiations linguistiques diatopique, diachronique et diastratique est tout aussi important que leurs productions orales effectives. La conscience linguistique d'un individu peut s'exprimer de différentes manières et elle peut être : i) explicitée par des croyances, des savoirs (intuitifs), des opinions ou des jugements linguistiques des locuteurs (on parle de conscience linguistique explicite); ii) caractérisée par des représentations, des comportements ou des attitudes linguistiques des locuteurs (on parle alors de conscience linguistique implicite) (Terracini, 1963 ; Schlieben-Lange, 1971; Telmon, 2000 ; Iannàccaro, 2002). Par ailleurs, les perceptions se développent sur deux plans complémentaires : (a) le plan du linguiste et (b) celui du non-linguiste/locuteur. Les deux visions s'influencent et donnent lieu à une « transmission des savoirs » (Iannàccaro, 2002).

4 Les définitions des spécialistes à propos du domaine de recherche sont très variées, mais elles dépendent essentiellement des approches théoriques et méthodologiques adoptées. Nous choisissons ici de proposer celles formulées par les chercheurs italiens qui ont inspiré notre étude.

5 À l'Université de Turin, Telmon considère la DP comme une branche de la dialectologie dont le programme est l'étude de la perception des locuteurs à l'égard de chacun des phénomènes linguistiques qui les concerne, qu'elle soit relative à leur parler ou aux parlers des autres. Telmon ajoute que l'on peut aussi s'intéresser à la perception de la variation interne d'un parler ou à une variété qui compose le répertoire linguistique d'un locuteur :

[...] une branche de la dialectologie qui se fixe comme but l'étude de la perception des locuteurs envers chaque phénomène linguistique qui les concerne, qu'elle soit relative à leur parler ou aux parlers des autres; ou encore, qu'elle soit relative à la variation interne de leur parler ou à celle de leur répertoire [linguistique] [...] (Telmon, $2000: \mathrm{VI})^{1}$.

6 À l'Université de Milan, Iannàccaro \& Dell'Aquila pensent non seulement que la DP est une branche de la linguistique liée à la géographie :

[...] perceptual dialectology, [is] a branch of linguistics strictly connected with geography (Iannàccaro \& Dell'Aquila, $2001: 265$ ).

7 Mais aussi que la DP est une «discipline frontière » entre la sociolinguistique et la géolinguistique :

[a] 'border' discipline of both sociolinguistics and geolinguistics [...] perceptual dialectology - that is, the scientific evaluation of the speaker's opinion - can be viewed as a means of integrating these two perspectives. Its goal is the study of the geographical treatment of the variation of the language itself; in other words, it focuses on linguistic habits and notions of linguistic identification (Dell'Aquila \& Iannàccaro, $2001:$ :265-266).

On retiendra que les «écoles » italiennes, dans ce secteur, adoptent conjointement les approches sociolinguistiques et géolinguistiques (Telmon, 2000 ; Iannàccaro, 2000, 2002) et, qu'en Italie, la DP est considérée, soit comme une branche/sous-branche de la linguistique (i.e. "branche de la dialectologie »), soit comme une " discipline frontière " entre deux ou plus secteurs disciplinaires (i.e. " géographie », "géolinguistique » et «sociolinguistique »). Les trois chercheurs sont d'accord sur l'objet d'étude de la DP : l'évaluation scientifique des «perceptions " qu'ont les locuteurs sur la variation d'une 
langue qu'ils emploient, qui compose leur répertoire linguistique ou qu'ils entendent (Telmon, 2000 : VI-XII ; Iannàccaro, 2000 : 84-85 ; Iannàccaro \& Dell'Aquila, 2001).

\section{Pourquoi une dialectologie perceptuelle?}

9 Ce que les non-linguistes croient, à propos de la langue qu'ils utilisent, fait partie du folklore - la science des traditions, des coutumes, des croyances, des arts populaires étudiée en ethnographie et en anthropologie culturelle (Preston, 1987, 1989, 1999; Niedzielski \& Preston, 2000). On observe une interaction pertinente entre, d'un côté, les croyances et les pratiques populaires et, de l'autre, les connaissances scientifiques (Berruto, 2000 ; Dell'Aquila \& Iannàccaro, 2001 ; Iannàccaro, 2000, 2002 ; Telmon, 2000, 2005). Certains secteurs de la linguistique ont besoin des apports et des connaissances des non-linguistes pour analyser la dynamique linguistique et culturelle caractérisant une aire géographique quelconque (Preston, 1999, 2002 ; Long \& Preston, 2003).

10 Un des objectifs de la DP est de " [...] déterminer et de comprendre le vécu sociolinguistique d'une communauté donnée ainsi que d'expliquer bon nombre de phénomènes, parmi lesquels ceux de la variation et du changement linguistiques. » (Berruto, $2000: 342)^{2}$.

11 Ainsi, la DP naît comme un domaine de recherche interdisciplinaire favorisant les connexions entre différents secteurs de la linguistique, mais aussi des Sciences Humaines et Sociales, tels que la géographie, l'anthropologie, l'ethnographie, la dialectologie, la sociolinguistique, la sociologie, la psycholinguistique et la psychologie sociale. Mais elle présente aussi un réel intérêt pour les acteurs d'autres disciplines de terrain tels que les enseignants de langues, notamment en didactique des langues (Preston, 2002 ; Niedzielski \& Preston, 2000). En Italie, les chercheurs des universités de Turin et de Milan emploient la DP pour résoudre des problématiques liées à la sauvegarde des minorités linguistiques et à la planification linguistique (Telmon, 1988b, 2000, 2005 ; Iannàccaro, 2000, 2002).

\section{3. «Méthodes croisées »}

12 L'approche adoptée dans notre travail de thèse s'inspire principalement des travaux de Telmon $(1988 b, 2000,2005)$ et de Iannàccaro $(2000,2002)$ menés en Italie et en Europe, depuis les années 1990 et 2000. Les chercheurs, dans la continuité des études de Terracini (entre autres, Terracini, 1963), ont aussi adopté des méthodes de collecte de données issues des études néerlandaises (Preston, 1999; Weijnen, 2003), japonaises (Grootaers, 1859, 1964 ; Mase, 1964) et américaines (Preston, 1989 ; Niedezelski \& Preston, 2000 ; Long \& Preston). Par le biais de ces techniques, il est possible de recueillir des données de nature différente. Dans notre cas, les enquêtes de terrain nous ont permis de recueillir des données distribuées sur deux niveaux d'analyse :

- un niveau "descriptif/objectif», constitué de données descriptives (lexicales et phonétiques) sur les parlers enquêtés - recueillies selon une approche de géolinguistique descriptive ;

- un niveau "perceptuel/subjectif ", composé des donnés «subjectives " sur la conscience linguistique des locuteurs - récoltées selon les approches italiennes, japonaises et américaines précédemment citées. 
13 Nous disposons, à la fois, d'enregistrements oraux de données «objectives » et/ou «subjectives » et des croquis cartographiques dessinés par les informateurs, sur des phénomènes linguistiques qu'ils ont perçus.

14 Par ailleurs, en DP, les méthodes d'analyse et de traitement des données diffèrent d'une " école » à l'autre et découlent du type d'approche théorique sous-jacente. D'une manière générale, après avoir analysé les deux blocs de données "objectives » et « subjectives " séparément, l'on procède à une analyse comparative. Ceci permet de se rendre compte si la réalité linguistique observée par le linguiste rejoint ou non la perception des locuteurs concernant le panorama linguistique qu'ils perçoivent et dans lequel ils évoluent.

Nos données "objectives" ont été collectées et analysées dans une perspective de géolinguistique descriptive. Pour l'interprétation des données "perceptuelles » nous avons eu recours aux approches et techniques à la fois de la sociolinguistique, de la psycholinguistique et de l'ethnolinguistique. La comparaison entre les deux groupes de données a tenu compte des dynamiques spécifiques de variation linguistique de l'aire italienne (Berruto, 1995; D’Agostino, 2007): pour cela nous avons été attentive aux niveaux de variation diatopique, diastratique, diachronique et diaphasique. Nous avons, aussi, réalisé des analyses quantitatives et qualitatives, cartographiques et géostatistiques : ce qui nous a permis de croiser un plus grand nombre de données de nature différente. Ces méthodes de collecte et de traitement des données sont propres à la DP et sont employées à la fois en géolinguistique, en sociolinguistique, mais aussi en géographie et en histoire.

\section{Recueil des données}

Les données ont été récoltées de 2007 à 2011, dans dix communautés linguistiques du Piémont occidental italien, situées dans la Province de Turin (Italie du nord-ouest). Les localités se trouvent, notamment, à l'entrée des Val Cluson, Val Germanasca (Val Saint Martin) et Val Pellice (Val Pellis) - mieux connus sous le nom de « vallées vaudoises », en Italie. Il s'agit de: Pignerol, San Secondo de Pignerol, Prarostino, Porte, San Germano Chisone, Frossasco, Roletto, Osasco, Airali et Bibiana. La population par localité varie d'un minimum de 1.124 habitants (Porte) à un maximum de 36.180 habitants (Pignerol). La distance entre chaque localité varie de $2 \mathrm{~km}$ à $15 \mathrm{~km}$.

Dans cette aire, la présence de différentes variétés « officielles » et « non-officielles » rend compte d'une situation linguistique complexe ${ }^{3}$ (Berruto, 1995 ; Cornagliotti, 1997). En effet, les spécialistes italiens constatent la présence, dans l'aire, de quatre codes linguistiques : l'italien (régional), le haut-piémontais (gallo-italien), l'occitan alpin (gallo-roman) et le français (vaudois). Les trois premiers codes semblent être en situation de "plurilinguisme " avec diglossie, alors que le français n'entretient aucun rapport diglossique avec les autres codes (Telmon, 1988 ; Cornagliotti, 1997 ; Cini \& Regis, 2000).

Nous nous concentrons sur les résultats obtenus pour 32 locuteurs, dont 18 femmes et 14 hommes ${ }^{4}$. Leur âge se distribue sur trois tranches différentes : a) 18-30 ans ; b) 40-60 ans ; c) plus de 70 ans. Ceci pour respecter les caractéristiques propres au panorama 
sociolinguistique italien. Afin de s'assurer de leur dialectophonie active ou passive, chaque informateur devait résider dans une des localités depuis au mois dix ans.

Ainsi, nous avons mis en place deux questionnaires et deux travaux de cartographie, soumis oralement aux informateurs. Par ordre, nous avons:

- 1. un questionnaire descriptif, nécessaire pour recueillir les données objectives (lexique, phonétique et morphologie);

- 2. un questionnaire perceptuel permettant de collecter les données sur la conscience linguistique des locuteurs - inspiré des questionnaires de Telmon (2000) et Iannàccaro (2002);

- 3. un travail de cartographie - initialement suggéré dans les études de Weijnen (2003), puis repris par Grootaers (1964) et Mase (1964).

Les techniques d'entretien ont été adaptées aux travaux mis en place. Nous avons privilégié un entretien semi-directif visant à recueillir des données lexicales, en proposant aux informateurs de décrire des images et des photos, afin de ne pas biaiser la vision que l'informateur a de "son propre monde». Passé ce premier entretien, nous avons demandé aux locuteurs de se focaliser sur quelques mots, qu'ils ont prononcés de façon aléatoire, afin de collecter les détails phonétiques sur les parlers. Pour finir, nous avons choisi d'alterner les techniques propres aux entretiens semi-directifs et libres pour les enquêtes perceptuelles, afin de sonder plus facilement la conscience linguistique des locuteurs et de les laisser s'exprimer librement.

21 L'objectif final est d'identifier les marqueurs de l'identité linguistique des locuteurs dans un panorama où les minorités linguistiques (non-officielles) - variétés d'occitan cohabitent avec les parlers locaux majoritaires (haut-piémontais), la langue nationale (l' italien régional piémontais), et le français utilisé dans les domaine familial ou religieux (rares cas).

\subsection{Questionnaire descriptif : quelques données}

22 Le questionnaire descriptif que nous avons soumis aux informateurs présentait de nombreuses entrées (plus d'une centaine) nous permettant d'enquêter sur la structure linguistique des parlers locaux à plusieurs niveaux : lexical et phonétique. Pour le lexique, nous nous sommes limitée à quelques champs lexicaux : les animaux d'élevage, la maison, le corps humain, la nature, le temps et les aliments (290 entrées). Nous avons, aussi, établi un inventaire phonétique vocalique propre à chaque variété locale. L'examen de ces traits (lexicaux et phonétiques) nous a donné des indices sur les dynamiques de variation linguistique de l'aire en question : celle-ci s'articule sous forme de gradata linguistiques ${ }^{5}$, où les extrema sont caractérisés par le haut-piémontais et l'occitan (à ce propos $c f$. Stehl, 1988). Les extrema sont orientés, respectivement, dans un sens géographique allant du sud-est au nord-ouest.

Dans la figure 1 qui suit, nous montrons un exemple de traitement de données phonétiques en géolinguistique descriptive qui illustre bien ce phénomène de gradatum linguistique. Il s'agit de l'analyse des aboutissants du latin CAPRA(M), dans les dix variétés enquêtées. Les points linguistiques étudiés dans cet article sont représentés, sur la carte, par un sigle (par exemple : $\mathrm{Bi}, \mathrm{Ai}, \mathrm{Os} . .$. ). 


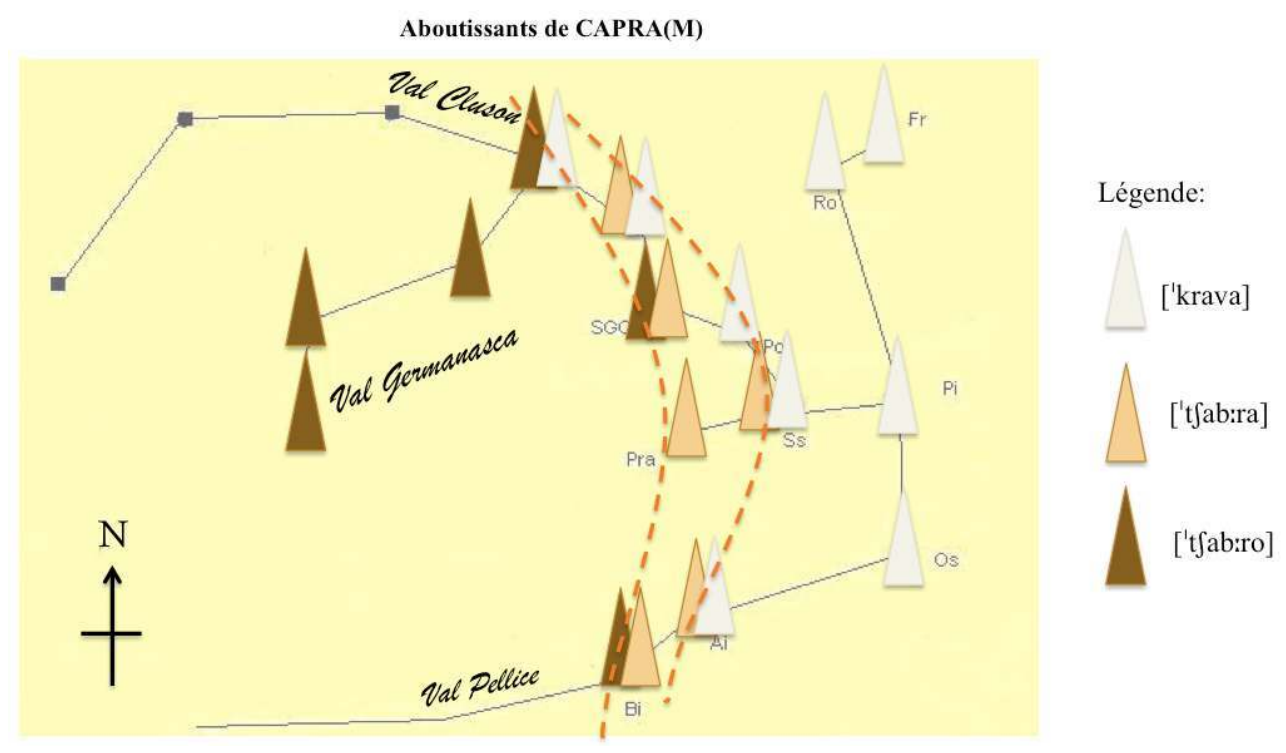

Figure 1 : traitement cartographique de données phonétiques - aboutissants de CAPRA(M)

Sur cette carte, nous distinguons trois réalisations phonétiques pour un même lemme ${ }^{6}$ : (a) ['krava]; (b) ['tfab:ra]; (c) ['t $\mathrm{t} a \mathrm{ab}: \mathrm{ro}$ ]. Comme nous pouvons le constater, nombreuses sont les localités où coexistent deux occurrences ${ }^{7}$ pour le même référent, "chèvre ". L'occurrence haut-piémontaise ['krava] se caractérise par le maintient du [k] vélaire initial et la métathèse de la vibrante (remontée en syllabe initiale). En revanche, dans la forme occitane ['tfab:ro] on note une palatalisation $\mathrm{du}[\mathrm{k}]>[\mathrm{t} \mathrm{t}]$ ainsi que la présence $\mathrm{du}$ morphème grammatical du genre féminin [-o]. Nous remarquons, ici, que le contact linguistique entre les variétés gallo-romanes et les variétés gallo-italiennes locales a produit une forme phonétique ['t $t$ ab:ra] à mi-chemin entre les deux formes précédentes ${ }^{8}:$ elle est formée par un radical occitan ['t $f a b: r-]$ auquel s'est 'greffé' le morphème grammatical [a], marque du genre féminin des variétés gallo-italiennes (italien régional et variétés hautpiémontaises). Ceci rend compte de ce que les spécialistes appellent le phénomène d' italianisation des parlers locaux occitans au contact de l'italien utilisé dans la plaine turinoise et/ou des parlers haut-piémontais (Telmon, 1988b).

Les isoglosses, représentées en pointillé sur la carte, illustrent un diasystème de gradata qui caractérise notre aire linguistique (Telmon, 1988a ; Stehl, 1988). Plus on s'éloigne de la plaine (sud-est de la carte), plus les variétés locales piémontaises se démarquent les unes des autres, structurellement, jusqu'à ce que "graduellement" l'on se rapproche partiellement ou totalement des caractéristiques structurelles des variétés occitanes alpines (nord-ouest de la carte).

\subsection{Questionnaire perceptuel : données sur la conscience linguistique}

Le questionnaire perceptuel vise à enquêter sur la perception qu'ont les locuteurs du panorama linguistique dans lequel ils vivent, ainsi que sur la perception qu'ils ont de l'usage des codes et de leurs habitudes linguistiques: ce qui compose la conscience linguistique des locuteurs. On collecte, alors, les jugements, les opinions, les savoirs et les sentiments linguistiques que les informateurs peuvent avoir sur leur propre variété linguistique ou celles qui les entourent. 
27 Notre questionnaire présente 62 points et il est organisé en six sections différentes : a) informations sociolinguistiques sur le locuteur ; b) perception des domaines d'usage de la variété locale et de l'italien régional ; c) perception de la variété locale et de ses frontières ; d) perception de l'italien régional et de ses frontières; e) perception conjointe des deux (variété locale et italien régional); d) questions relatives aux travaux de cartographie. Les six sections tiennent évidemment compte des quatre niveaux linguistiques variationnels déjà évoqués : diatopique, diastratique, diachronique et diaphasique.

Ces données nous permettent d'observer d'éventuelles correspondances entre les attitudes et les comportements linguistiques déclarés par les locuteurs dans les entretiens et ce qui se réalise dans leur quotidien. En effet, nous avons enregistré nos informateurs pendant leurs interactions verbales tout au long de leurs activités, comme par exemple dans le milieu familial, dans les lieux publics (poste, bars, places publiques) et dans le domaine religieux.

\subsection{Travaux de cartographie}

Les travaux de "cartographie perceptuelle" donnent lieu à ce qu'on appelle plus communément des cartes-mentales (ou mental-maps). Il s'agit de croquis cartographiques, effectués par nos informateurs, qui permettent de comprendre la façon dont ils perçoivent la variation linguistique dans l'aire géographique étudiée - on appelle cela la "perception des frontières linguistiques » ou les «frontières linguistiques subjectives/ perçues".

30 Le premier travail de cartographie que nous avons développé est inspiré des études géolinguistiques néerlandaises des années 1930, menées par Weijnen (Preston, 1999 : iiixxx ; Weijnen, 2003). Dans « la cartographie little-arrows », les informateurs représentent, sur un support cartographique, des similitudes linguistiques entre parlers en tenant compte d'un modèle de référence qui reporte, de manière schématique, les points du réseau global d'enquête. La tâche qui leur est demandée est la suivante: "Dessinez une flèche entre deux localités si vous pensez qu'il y a une similarité entre les variétés locales. Le sens de la flèche indique : 'le parler X ressemble au parler Y'». Dans la figure 2 cidessous, nous avons reproduit le schéma little-arrows dessiné par une informatrice d'Osasco, Marina. 


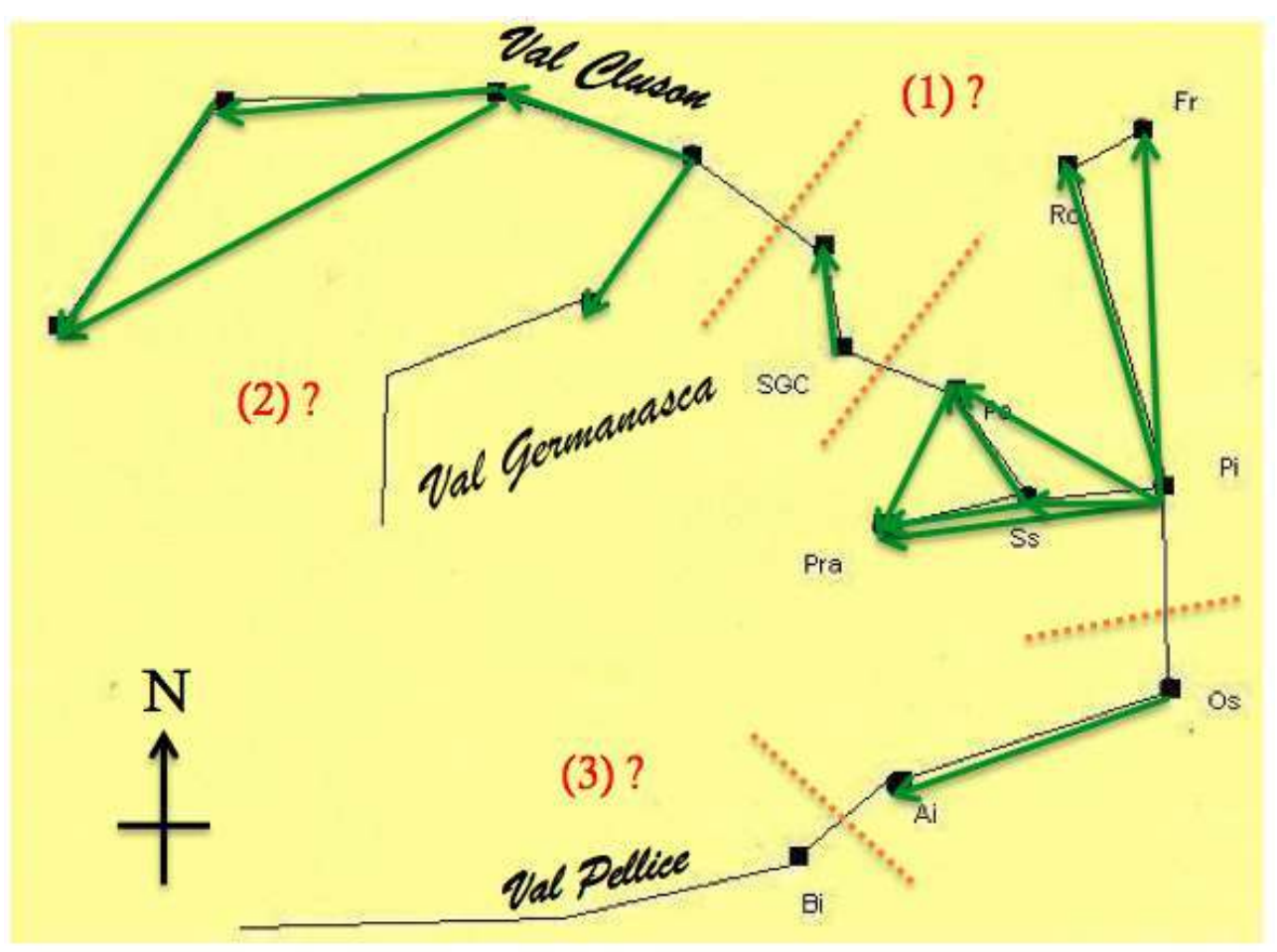

Figure 2 : schéma reproduisant la carte little-arrows de Marina (Osasco) apparente, ce type de cartographie soulève un certain nombre de problèmes. Tout d'abord, comment expliquer que les similitudes entre parlers ne soient pas réciproques pour notre informatrice? Nous savons que les flèches unidirectionnelles indiquent le sens de similitude : par exemple, Marina affirme que le parler de Pignerol (« Pi » sur la carte) ressemble au parler de Frossasco ( Fr » sur la carte) et non l'inverse. Pourquoi Marina at-elle dessiné une seule flèche dans ce sens et non pas une flèche allant de « Fr » à « $\mathrm{Pi}$ »? Lorsqu'on lui pose la question, Marina ne sait pas nous expliquer pourquoi et elle ne nous donne pas de réponse précise : nous rencontrons, alors, un premier problème. Ensuite, nous remarquons autre chose: comment traiter les zones marquées (1), (2) et (3) sur la carte ? Doivent-elles être considérées, aussi, comme des frontières perceptuelles, même si elles ne font pas partie de notre réseau d'enquête ? En conclusion, la cartographie littlearrows soulève un certain nombre de questionnements sur les limites méthodologique et analytique de ce type de travail.

Ainsi, nous avons soumis aux informateurs un second type de cartographie qui nous a permis de procéder à des interprétations beaucoup plus claires à propos des frontières linguistiques "perçues». Nous faisons notamment allusion à la cartographie degree-ofdifference, imaginée par les géolinguistes Grootaers et Mase, au Japon (Grootaers, 1959, 1964; Mase, 1964). Il s'agit d'un procédé cartographique d'étiquetage de variétés linguistiques par degrés de similitudes et de différences perçues. La tâche que nous avons soumis aux informateurs est la suivante : « Numérotez de 1 à 4 les parlers représentés par des points sur la carte : notez 1 si les parler sont IDENTIQUES au vôtre, 2 s'ils sont QUASI IDENTIQUES au vôtre, 3 s'ils sont DIFFERENTS du vôtre, 4 s'ils sont TOTALEMENT DIFFÉRENTS ou INCONNUS par rapport au vôtre. Attribuez le chiffre 1 à votre parler et numérotez ceux qui l'entourent. ». Dans la figure 3 qui suit, nous avons simplement 
retravaillé la carte d'une informatrice résidant à San Germano (Marina) en octroyant à chaque numéro une couleur différente, pour obtenir un meilleur rendu cartographique et visuel.

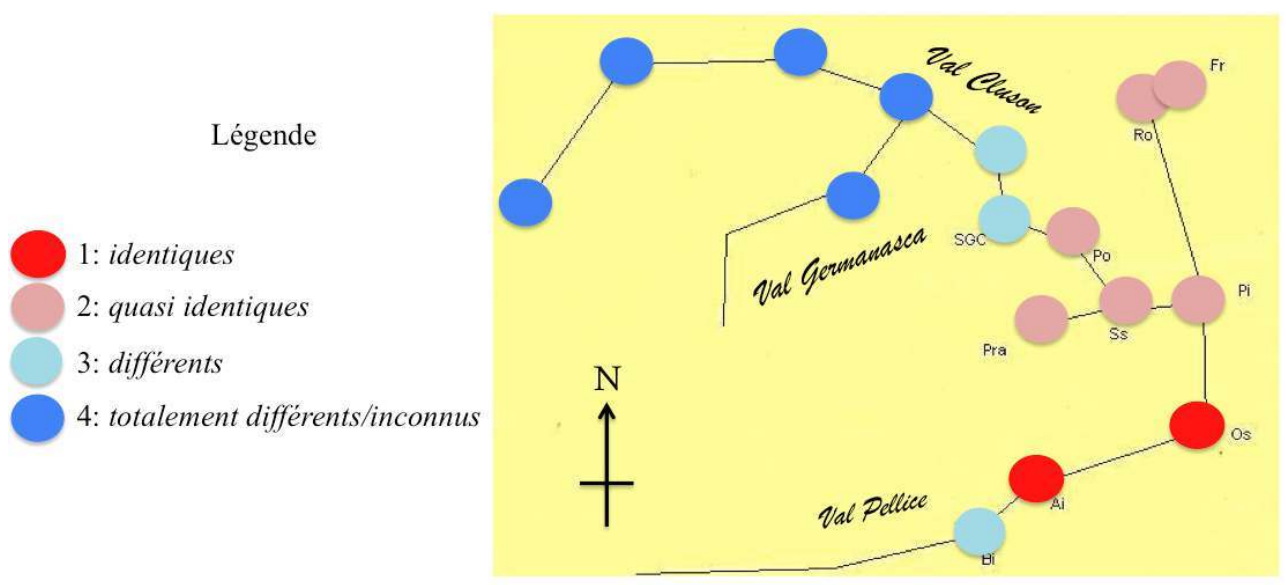

Figure 3 : schéma reproduisant la carte degree-of-difference de Marina (Osasco)

Cette "gradation subjective » des parlers paraît plus claire : plus on s'éloigne du parler d'Osasco plus les variétés linguistiques sont perçues, par Marina, comme étant différentes structurellement par rapport au parler de référence qui est le sien. L'étiquetage perceptuel et cartographique, degree-of-difference, a été volontairement choisi et appliqué à l'aire étudiée car il modélise la distance structurelle existante entre les variétés locales, caractérisée par des gradata linguistiques. Ici, les points linguistiques qui ne sont pas étiquetés dans la cartographie little-arrows, apparaissent plus distinctement dans ce type de classification perceptuelle. C'est une tendance générale observée chez $70 \%$ de nos informateurs : les résultats cartographiques du type degree-of-difference sont plus probants que ceux issus d'une représentation en little-arrows.

Ce type de carte nous permet d'analyser les choix de "similarité » ou de "différence » entre parlers effectués par les informateurs. En effet, chaque locuteur commente oralement son propre travail cartographique. En analysant leurs réponses, plusieurs tendances de "catégorisation subjective» des frontières linguistiques nous sont apparues. Tout d'abord, au premier niveau de différenciation linguistique (qui correspond à l'étiquetage " les parlers quasi identiques au vôtre »), il semblerait que les informateurs aient retenu les traits phonétiques, comme principal caractère discriminant dans l'identification et la reconnaissance des parlers. Les locuteurs nous expliquent qu'ils entendent/perçoivent parfois une différence dans la prononciation d'un même mot entre leur parler et les variétés voisines : cela peut se caractériser, par exemple, par un degré d'aperture et de fermeture des voyelles différent ${ }^{9}$. La perception de ces différentes réalisations leur permet de reconnaitre un dialecte par rapport à un autre. Au second niveau de catégorisation/représentation subjective (qui correspond à l'étiquetage «les parlers différents du vôtre »), les locuteurs se focalisent essentiellement sur des différences lexicales et/ou morphologiques: ces différences seraient, selon eux, dues à une faible compréhension (voire une incompréhension) de la variété qu'ils entendent. En choisissant l'étiquette « les parler totalement différents/inconnus par rapport au vôtre ", les informateurs mettent en avant des différences structurales « importantes/très grandes " entre dialectes : à leur avis, ces différences empêcheraient « une compréhension générale du discours » en agissant simultanément sur différents niveaux structuraux. À cet égard, 
les informateurs parlent de "syntaxe très compliquée et incompréhensible " mêlée à " une intonation indéchiffrable » et/ou " inconnue ", à laquelle s'ajouteraient " des mots jamais entendus ». Par exemple, un de nos locuteurs piémontais affirme ceci : (traduction) « Ne pas comprendre un mot dans une phrase c'est une chose, parfois ça me fait sourire parce que je le trouve drôle [le mot] (rires) quand je parle avec des gens de la vallée (pause) mais si tu changes tous les mots et tu rajoutes en plus le phrasé impossible à comprendre, là, c'est fini (pause) je me perds (rires) un peu comme en patois [occitan], quand je vais à là-haut [i.e. à Pragelato] (rires) ».

\section{Bilan : une méthodologie « en construction »}

Nous avons vu que d'un point de vue théorique et méthodologique les études de DP peuvent réunir plusieurs approches et techniques empruntées à la géolinguistique, à la sociolinguistique, mais aussi à la géographie et à l'histoire, à l'ethno-anthropologie et/ou à la psychologie sociale. Ceci parce que, pour répondre à une problématique donnée, la linguistique et ses sous-disciplines peuvent s'affranchir de toutes les connaissances développées aussi bien dans les sciences exactes que dans les sciences humaines et sociales. C'est donc une caractéristique intrinsèque de la linguistique, et de ses sousbranches que de se servir de l'apport d'autres disciplines pour résoudre une problématique donnée. La DP, tout particulièrement, peut être vue comme une interface entre la linguistique de terrain et la linguistique descriptive des éléments constitutifs des langues.

Mais la DP est un domaine de recherche non consolidé, relativement novateur et récent, et en devenir. En se servant de ce que pensent les non-linguistes à propos de la langue, le linguiste touche au domaine de la conscience. La conscience linguistique, qui fait partie de cette dernière, se développe et prend forme "dans le locuteur ", mais aussi, dans une transmission de savoirs "avec le locuteur", c'est-à-dire dans un échange entre le spécialiste et le non-spécialiste ( $c f$. Iannàccaro, 2000, 2002). Ainsi, les approches adoptées dans ce secteur sont étroitement liées à ces deux aspects. C'est pourquoi il paraît nécessaire de respecter et appliquer toujours le même type de formulation aux entrées des questionnaires perceptuels : la manière dont le linguiste formule une question influe sur la réponse fournie par le locuteur. Et la formulation des questions requiert, de la part du linguiste, un travail méticuleux en amont, ainsi qu'un degré d'attention constant tout au long de l'entretien.

La nature composite et le grand nombre de données récoltées augmentent la complexité d'interprétation et d'observation linguistiques; cela se résout facilement par une sélection d'échantillons plus restreinte. Par ailleurs, selon les spécialistes (Telmon, 2000 ; Iannàccaro, 2002), il conviendrait d'utiliser les données « perceptuelles » avec parcimonie puisqu'elles seraient, de par leur nature, « contradictoires ». Étant donné la multitude et la diversité des données récoltées dans ce type d'études, les chercheurs Dell'Aquila et Iannàccaro ont démontré que l'analyse et le traitement géostatistique des données ${ }^{10}$ seraient appropriés et permettraient d'obtenir des résultats plus probants (Iannàccaro \& Dell'Aquila, 2001 ; Dell'Aquila \& Iannàccaro, 2006).

Par ailleurs, nous avons remarqué que les cartes-mentales du type little-arrows, malgré leur originalité, présentent quelques problèmes évidents d'interprétation ; alors que la cartographie degree-of-difference permet une analyse plus précise des frontières linguistiques perçues. 
En conclusion, l'observation des «critères subjectifs » qui définissent la variation linguistique chez les non-spécialistes représente un réel intérêt pour les linguistes. Ceci pour deux raisons qui consistent, d'abord, à comprendre si la perception du monde linguistique d'un non-spécialiste rejoint ou non la description effectuée par un scientifique, ensuite, à explorer le monde du locuteur pour mieux comprendre la langue qu'il emploie.

\section{BIBLIOGRAPHIE}

Berruto, G. (1995), Fondamenti di sociolinguistica, Roma-Bari : Laterza.

Berruto, G. (2000), « Sul significato della dialettologia percettiva per la linguistica e la sociolinguistica », in Cini, M. et Regis, C. (éds.) (2000) : 341-362.

Cini, M., Regis, C. (éds.) (2000), Atlante Linguistico ed etnografico del Piemonte Occidentale, Che cosa ne pensa oggi Chiaffredo Roux? Percorsi della dialettologia percezionale all'alba del nuovo millennio, Vol. 6 Atti del convegno internazionale Bardonecchia 25-27 maggio 2000 -, Università degli Studi di Torino dipartimento di Scienze del Linguaggio : Ed. dell'Orso.

Cornagliotti, A.(1997), « Italien - occitan », in Goeblh, H., Nelde, P.H., Stary, Z., Wölck, W. (éds.) (1997), Kontaktlinguistik - Contact Linguistics - Linguistique de contact, Volume II, Berlin-New York : Walter de Gruyter Ed., 1344-1349.

D’Agostino, M. (2007), Sociolinguistica dell'Italia contemporanea, Bologna : Il Mulino.

Dell'Aquila, V., Iannàccaro, G. (2006), Survey ladins. Usi linguistici nelle valli ladine, Trento : Ed. Regione Autonoma Trentino-Alto Adige / Südtirol.

Falkert, A. (2010), Le français acadien des Îles-de-la-Madeleine. Étude de variation phonétique, Paris : L'Harmattan.

Goeman, T. (1989), « Dialectes et jugements des locuteurs. Quelques remarques de méthode à propos d'une controverse », in Espaces Romans. Etudes de dialectologie et de géolinguistiques offertes à Gaston Tuallion, Vol. II, Grenoble : Ellug, 532-544.

Grootaers, W.A. (1959), " Origin and nature of the subjective boundaries of dialect ", in ORBIS n ${ }^{\circ}$ VIII/2, Louvain :Ed. Centre International de Dialectologie Générale, 355-384.

Grootaers, W.A. (1964), « La discussion autour des frontières dialectales subjectives », in ORBIS n • XIII/2, Louvain : Ed. Centre International de Dialectologie Générale, 380-398.

Iannàccaro, G. (2000), « La percezione del cambio linguistico nel parlante », in Cini, M., Regis, C. (éds.) (2000) : 81-107.

Iannàccaro, G. (2002), Il dialetto percepito. Sulla reazione dei parlanti di fronte al cambio linguistico, Tesi di Dottoratodell'Università di Firenze, Alessandria : Ed. dell'Orso.

Iannàccaro, G., Dell'Aquila, V. (2001), « Mapping languages from inside: notes on perceptual dialectology » [en ligne], Social \& Cultural Geography, Vol. 2 - No. 3, [page consultée en 2010] disponible à l'adresse suivante : 
http://www1.geo.ntnu.edu.tw/ moise/Data/Books/Social/09\%20other/mapping\%20languages\% 20from\%20inside\%20notes\%20on\%20perceptual\%20dialectology.pdf

Léonard, J.L. (1987), Démarcation linguistique. Conscience de la variation dialectale et dialectologie du locuteur, sous la direction de Bouvier, J.-C., Aix-en-Provence : Faculté de Lettres d'Aix-enProvence.

Léonard, J.L. (2003), « Microcosmic perceptual dialectology and the consequences of extended linguistic awarness. A case study of Noirmoutier Island (France) ", in Long, D. et Preston, D. R. (2003) : 221-248.

Long, D., Preston, D.R. (éds.) (2003), Handbook of Perceptual Dialectology. Volume II, AmsterdamPhiladelphia : Benjamins.

Mase, Y. (1964), « Une nouvelle tentative pour tracer les frontières subjectives des dialectes », in ORBIS $n^{\circ}$ XIII/2, Louvain : Ed. Centre International de Dialectologie Générale, 357-379.

Niedzielski, N. A., Preston, D. R. (2000), Folk Linguistics, Berlin-New York : Werner Winter Editor. Preston, D.R. (1989), Perceptual dialectology: nonlinguists' views of areal linguistics, Dodrecht : Foris. Preston, D.R. (éd.) (1999), Handbook of Perceptual Dialectology. Volume I, Amsterdam-Philadelphia : Benjamins.

Preston, D.R. (2002), « Down and Out in Perceptual Dialectology », in D’Agostino, M. (éd.) (2002), Percezione dello spazio e spazio della percezione. La variazione linguistica fra nuovi e vecchi strumenti di analisi, Vol. 10, Palermo : Dipartimento di Scienze Filologiche e Linguistiche (Facoltà di Lettere e Filosofia), 11-38.

Pustka, E. (2007), Phonologie et variétés en contact. Aveyronnais et Guadeloupéens à Paris, Tübingen : Niemeyer.

Schlieben-Lange, B. (1971) « La conscience linguistique des occitans », in Revue de Linguistique Romane $n^{\circ} 35$ : 298-303.

Stehl, T. (1988), « Les concepts de continuum et de gradatum dans la linguistique variationnelle ", in Kremer, D. (éd.), Actes du XVIIIe Congrès International de Linguistique et de Philologie Romanes. Université de Trèves (Trier) 1986, Bd. V: Section IV. Linguistique pragmatique et linguistique sociolinguistique, Tübingen : Niemeyer 28-40.

Telmon, T. (1988a), « Italienisch: Arealinguistik II. Piemont », in Holtus, G., Metzeltin, M., Schmitt, C. (éds.) (1991), Lexikon der Romanistischen Linguistik, band IV, Tubingen : Max Niemeyer, 469-485.

Telmon, T. (1988b), « Il dialetto imbastardito. L'elemento straniero nella valutazione del parlante ", in Elementi stranieri nei dialetti italiani - Atti del XVI Convegno del C.S.D.I. - Voll. II,Ivrea 7-19 ottobre 1984, Pisa : Pacini, 1-10.

Telmon, T. (2000), « Le ragioni di un titolo », in Cini, M., Regis, C. (éds.) (2000) : V-XXXIV.

Telmon, T. (2005), « Una ricerca sulla percezione dei parlanti circa i rapporti tra italiano e dialetto », in Lo Piparo, F., Ruffino, G. (2005), Gli italiani e la lingua, Palermo : Sellerio Editore, 229-254.

Terracini, B. (1963), Lingua libera e libertà linguistica. Introduzione alla linguistica storica, Torino : Einaudi.

Weijnen, A. A. (2003), « On the value of subjective dialect boundaries », in Long, D., Preston, D.R. (éds.) (2003) : 131-262. 


\section{NOTES}

1. Cette traduction est la nôtre. Texte original: « [...] un ramo della dialettologia che funzionalmente e programmaticamente si prefigge di studiare la percezione dei parlanti nei confronti di ogni fatto linguistico che li concerne, sia esso relativo alla propria parlata o a quella degli altri ; o sia relativo alla variazione interna della propria parlata o a quella del proprio repertorio [...]» (Telmon, $2000: \mathrm{VI})$.

2. Cette traduction est la nôtre. Texte original : « [...] determinare e capire l'articolazione e il vissuto sociolinguistico della comunità e per spiegare molti fatti e fenomeni linguistici, fra cui in primo piano quelli della variazione e del mutamento linguistico. » (Berruto, 2000 : 342).

3. Le panorama sociolinguistique de la péninsule italienne est caractérisé par un type de diglossie que Berruto (1995) appelle dilalie (i.e. une séparation fonctionnelle des codes linguistiques qui composent le répertoire des locuteurs).

4. Les femmes sont surreprésentées parmi nos informateurs dans les communautés linguistiques choisies, simplement parce qu'il était plus facile pour nous d'engager un échange linguistique avec une femme, plutôt qu'avec un homme. Les communautés enquêtées se trouvent, principalement, en bordure de plaine et sur les flancs de montagne. Les activités professionnelles des informateurs varient en fonction de leur lieu de résidence, qu'il s'agisse d'un village ou qu'il s'agisse de la ville de Pignerol.

5. Nous renvoyons aux propos formulés par Stehl (1988) sur les notions de gradatum et de continuum en linguistique variationnelle. L'auteur conçoit la « zone de contact comme un diasystème de gradata » où « le concept d'une gradation de variétés discrètes qui se situent entre les extrêmes des deux langues en contact correspond aussi bien à la réalité de la zone de variation pragmatique qu'à celle de la zone d'interférence linguistique [...]» (Stehl, 1988 : 30).

6. Sur la carte, certains points linguistiques présentent deux aboutissants phonétiques du mot «chèvre »: cela, soit parce que les 3 locuteurs d'un même point n'employaient pas la même forme, soit parce qu'un même locuteur utilisait deux formes, indistinctement, lors des entretiens. 7. En effet, cela dépend non seulement de la configuration linguistique de l'aire, mais aussi des informateurs qui emploient une ou deux désignations, au choix, et qui nous en ont fait part pendant les entretiens.

8. À savoir que de Villar Perosa à Perosa Argentina, subsistent (nous l'avons remarqué chez certains locuteurs âgés) des formes du type ['t $t a b: r ə]$ où l'élément vocalique final est parfois centralisé (schwa) ou peu prononcé. Nous avons jugé bon de ne pas les représenter sur la carte, pour éviter de surcharger l'image.

9. Notamment, ils perçoivent une différence entre les deux voyelles antérieures, mi-fermée et mi-ouverte, [e] et [ $\varepsilon]$; ainsi qu'entre les voyelles postérieures, mi-fermée et mi-ouverte, [o] et [o]. 10. Par l'usage de logiciels spécifiques de traitement cartographique ou par le biais de la géomatique appliquée.

\section{RÉSUMÉS}

Actuellement, les recherches dans le domaine de la dialectologie perceptuelle (DP) sont très variées, mais elles s'intéressent globalement à l'étude de la conscience linguistique des locuteurs, ou encore, 
aux perceptions qu'ont les locuteurs de leurs propres variétés linguistiques et de celles avec lesquelles ils entrent en contact. En DP ce que les locuteurs croient et perçoivent quant à la différentiation linguistique diatopique est tout aussi important que leurs productions linguistiques effectives. L'objectif est de comprendre et de déterminer le vécu sociolinguistique d'une communauté donnée ainsi que d'expliquer bon nombre de phénomènes, parmi lesquels ceux de la variation et du changement linguistiques. C'est dans cette optique que nous voudrions présenter des données de DP récoltées dans dix localités du Piémont occidental italien (province de Turin). Nous proposons d'examiner nos résultats sur la base des méthodologies développées dans ce secteur d'études qui permettent de collecter des données linguistiques de différente nature, à savoir: a) les données descriptives - lexicales, phonétiques, morphologiques et/ou syntaxiques ; b) les données perceptuelles - jugements, opinions, sentiments, comportements et attitudes linguistiques - que les informateurs développent dans une aire linguistique.

Although research conducted within the framework of Perceptual Dialectology (PD) varies considerably, its primary focus is on the study of speakers' linguistic consciousness and linguistic awareness, in particular speakers' perceptions of the language and language varieties that are spoken around them. For PD what the speakers perceive and think about the linguistic diatopic variation is as important as real speech productions. The aim of this field of study is to understand and to check a community's sociolinguistic habits and to explain phenomena like linguistic variation and linguistic change. For these reasons, we present PD data collected in ten villages of the western Piedmont, in the northwest of Italy. We provide an analysis of our results inspired by the PD studies and its methodologies. Adopting this approach, we gathered a substantial amount of data as : a) descriptive data, in order to analyse the phonetic, lexical and morphological structures of linguistic varieties; b) perceptual data on linguistic consciousness and awarenes like opinions, judgments, feelings, behaviours and attitudes.

\title{
INDEX
}

Mots-clés : Linguistique de terrain, dialectologie perceptuelle, variation linguistique, contact de langues, parlers à tradition orale, sociolinguistique, géolinguistique.

Keywords : Language in contact, perceptual dialectology, sociolinguistics, linguistic variation, oral varieties, geolinguistics.

\author{
AUTEUR \\ SILVIA GALLY \\ Univ. Grenoble Alpes [GIPSA-lab, UMR 5216] et Université de Turin (Italie) \\ silvia.gally@gipsa-lab.grenoble-inp.fr, silviagally.sg@gmail.com
}

